\title{
Methotrexate as a Treatment of Palmoplantar Lichen Planus
}

\author{
Morgan Covington, MD; Carly Roman, MD; Farah R. Abdulla, MD
}

\section{PRACTICE POINTS}

- Palmoplantar lichen planus $(L P)$ is a rare variant of $L P$ that is resistant to most treatments.

- Methotrexate may be a cost-effective option in patients who cannot tolerate systemic retinoids.

To the Editor:

Palmoplantar lichen planus (LP) is an uncommon variant of LP that involves the palms and soles. The prevalence of LP is approximately $0.1 \%$ to $2 \%$ in the general population. It can affect both mucosal and cutaneous surfaces. ${ }^{1}$ A study of 36 patients with LP showed that 25\% (9/36) had palmar and/or plantar involvement. ${ }^{2}$ Palmoplantar LP is more commonly found in men than women, with an average age of onset of 38 to 65 years. ${ }^{3}$ It tends to affect the soles more often than the palms, with the most common site being the plantar arch. Itching generally is the most common symptom reported. Lesions often resolve over a few months, but relapses can occur in 10\% to $29 \%$ of patients. ${ }^{2}$ The clinical morphology commonly is characterized as erythematous scaly plaques, hyperkeratotic plaques, or ulcerations. ${ }^{4}$ Due to its rare occurrence, palmoplantar LP often is misdiagnosed as psoriasis, eczematous dermatitis, tinea nigra, or secondary syphilis, making pathology extremely helpful in making the diagnosis. ${ }^{1}$ Darker skin types can obscure defining characteristics, further impeding a timely diagnosis. We describe a novel case of palmoplantar LP that was successfully treated with methotrexate.

A 38-year-old man with no notable medical history presented for dermatologic evaluation of a palmar and plantar rash of 4 months' duration. The rash was accompanied by intense burning pain and pruritus. Prior to presentation, he had been treated with multiple prednisone tapers starting at $40 \mathrm{mg}$ daily as well as combination therapy of a 2-week course of minocycline $100 \mathrm{mg}$ twice daily and clobetasol ointment twice daily for 4 months, with no notable improvement. Workup prior to presentation included a negative potassium hydroxide fungal preparation and a normal antinuclear antibody titer. A review of symptoms was negative for arthralgia, myalgia, photosensitivity, malar rash, Raynaud phenomenon, pleuritic pain, seizures, and psychosis.

Physical examination revealed focal areas of mildly thick, hyperkeratotic scale with desquamation on the plantar and palmar surfaces of the feet and hands. The underlying skin of the feet consisted of dyspigmented patches of dark brown and hypopigmented skin with erythema, profound scaling, and sparing of the internal plantar arches (Figure 1A). On the palms, thin hyperkeratotic plaques with desquamation and erythematous maceration of the surrounding skin were observed (Figure 2A). Thin white plaques of the posterior bilateral buccal mucosa were appreciated as well as an erosion that extended to the lower lip.

The differential diagnosis included LP, psoriasis, acquired palmoplantar keratoderma, and discoid lupus erythematosus. Tinea pedis and tinea manuum were less likely in the setting of a negative potassium hydroxide fungal preparation.

A biopsy of the lateral aspect of the left foot showed a cell-poor interface dermatitis that could resemble partially treated LP or a lichenoid hypersensitivity reaction (Figure 3). Given the clinical and pathologic findings, a diagnosis of palmoplantar LP was favored. The patient

From the Department of Dermatology, University of Chicago, Illinois.

The authors report no conflict of interest.

Correspondence: Morgan Covington, MD, 1950 W Polk St, Chicago, IL 60612 (morgan.covington@cookcountyhhs.org).

doi:10.12788/cutis.0068 
was on no medications or over-the-counter supplements prior to the appearance of the rash, making a lichenoid hypersensitivity rash less likely. The histology findings likely were muted, as they were done at the end of the prednisone taper.

Minocycline and clobetasol ointment were discontinued, and the prednisone taper was completed as originally prescribed. The patient was started on $25 \mathrm{mg}$ daily of acitretin for 4 weeks, then increased to $35 \mathrm{mg}$ daily. Notable improvement in the palmar and plantar lesions was noted after the initial 4 weeks of therapy; however, acitretin treatment was discontinued due to lack of adequate insurance coverage for the medication. The patient became symptomatic several weeks following acitretin cessation and was started on methotrexate $15 \mathrm{mg}$ weekly with triamcinolone acetonide paste $0.1 \%$ for the oral lesions. Once again, improvement was seen on both the palmar and plantar surfaces after 4 weeks of therapy (Figures 1B and 2B).

Evidence for treatment of palmoplantar LP is limited to a few case reports and case series. Documented treatments for palmoplantar LP include topical and systemic steroids, tazarotene, acitretin, and immunosuppressive medications. ${ }^{4}$ One case report described a patient who responded well to prednisone therapy $(1 \mathrm{mg} / \mathrm{kg}$ daily for 3 weeks, then reduced to $5 \mathrm{mg}$ daily). ${ }^{5}$ Another report described a patient who responded favorably to cyclosporine $3.5 \mathrm{mg} / \mathrm{kg}$ daily for 4 weeks, then tapered over another 4 weeks for a total of 8 weeks of treatment. ${ }^{4}$ Although the most common treatments described in the
FIGURE 1. A, Hyperkeratotic scaly lesions on the sole of the foot with sparing of the internal plantar arch. B, Improvement was seen after 4 weeks of methotrexate therapy.

FIGURE 2. A, Thin hyperkeratotic lesions on the palm with minimal scaling. B, Improvement was seen after 4 weeks of methotrexate therapy.
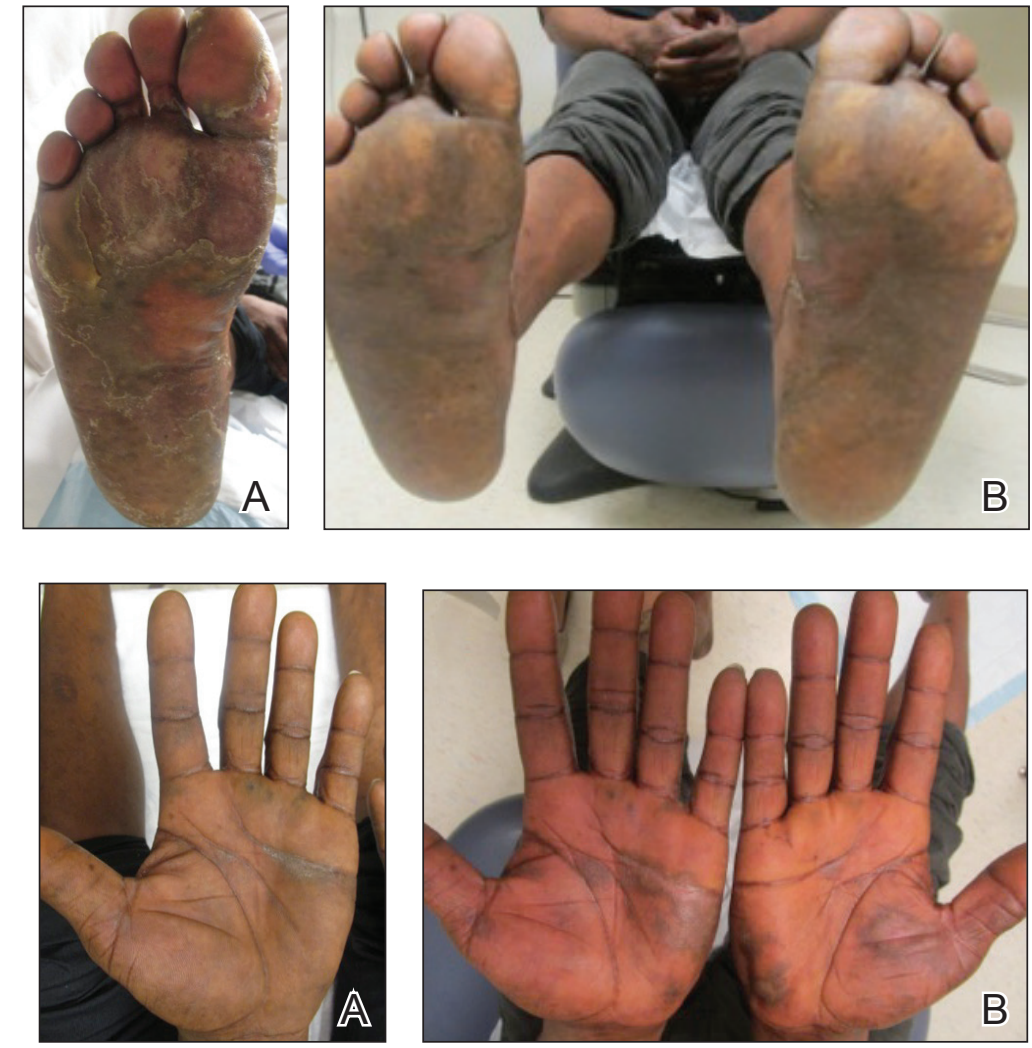

FIGURE 3. A, Histopathology of the lateral aspect of the left foot demonstrated hyperkeratosis (H\&E, original magnification $\times 40$ ). B, Cell-poor interface dermatitis also was seen (H\&E, original magnification $\times 100$ ).
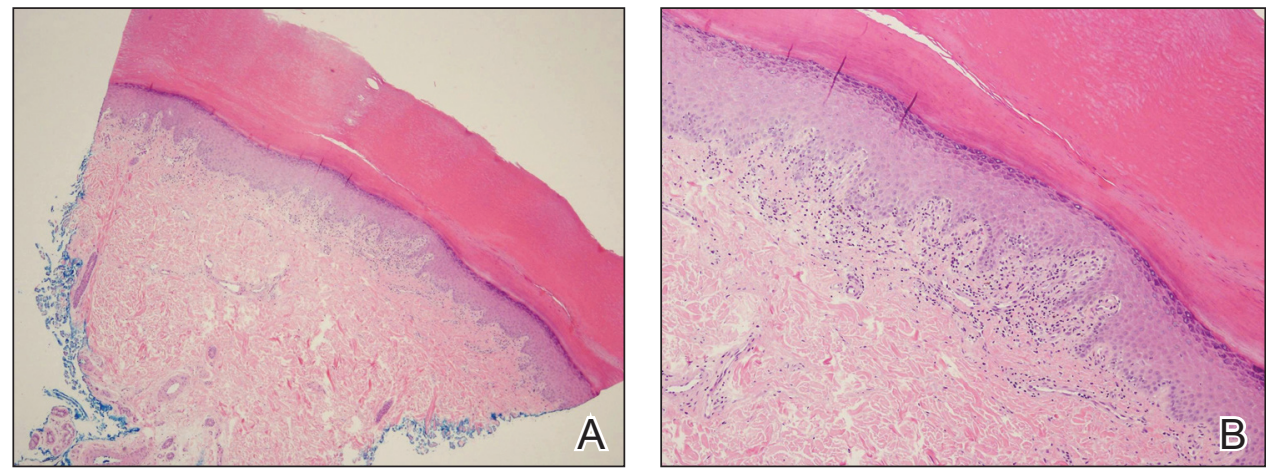
literature consist of acitretin as well as topical and systemic steroids, few have discussed the efficacy of methotrexate. In one study, acitretin did not result in clearance, but the patient saw profound improvement with methotrexate (titrated up to $25 \mathrm{mg}$ weekly) over 2 months. ${ }^{1}$

In our case, treatment with methotrexate was proven successful in a patient who responded to acitretin but was unable to afford treatment. This case highlights a rare variant of a common disease and the possibility of methotrexate as a cost-effective and useful treatment option for LP.

\section{REFERENCES}

1. Rieder E, Hale CS, Meehan SA, et al. Palmoplantar lichen planus. Dermatol Online J. 2015;20:13030/qt1vn9s55z.

2. Sánchez-Pérez J, Rios Buceta L, Fraga J, et al. Lichen planus with lesions on the palms and/or soles: prevalence and clinicopathological study of 36 patients. Br J Dermatol. 2000;142:310-314.

3. Gutte R, Khopkar U. Predominant palmoplantar lichen planus: a diagnostic challenge. Indian J Dermatol. 2014;59:343-347.

4. Karakatsanis G, Patsatsi A, Kastoridou C, et al Palmoplantar lichen planus with umbilicated papules: an atypical case with rapid therapeutic response to cyclosporin. J Eur Acad Dermatol Venereol. 2007;21:1006-1007.

5. Goucha S, Khaled A, Bennani Z, et al. Erosive lichen planus of the soles: Effective response to prednisone. Dermatol Ther. 2011;1:20-24. 